\title{
Using a reflection model for modeling the dynamic feedback path of digital hearing
} aids

Ma, Guilin; Gran, Fredrik; Jacobsen, Finn; Agerkvist, Finn T.

Published in:

Acoustical Society of America. Journal

Link to article, DOI:

$10.1121 / 1.3290989$

Publication date:

2010

Document Version

Publisher's PDF, also known as Version of record

Link back to DTU Orbit

Citation (APA):

Ma, G., Gran, F., Jacobsen, F., \& Agerkvist, F. T. (2010). Using a reflection model for modeling the dynamic feedback path of digital hearing aids. Acoustical Society of America. Journal, 127(3), 1458-1468.

https://doi.org/10.1121/1.3290989

\section{General rights}

Copyright and moral rights for the publications made accessible in the public portal are retained by the authors and/or other copyright owners and it is a condition of accessing publications that users recognise and abide by the legal requirements associated with these rights.

- Users may download and print one copy of any publication from the public portal for the purpose of private study or research.

- You may not further distribute the material or use it for any profit-making activity or commercial gain

- You may freely distribute the URL identifying the publication in the public portal 


\title{
Using a reflection model for modeling the dynamic feedback path of digital hearing aids ${ }^{\text {a) }}$
}

\author{
Guilin $\mathrm{Ma}^{\mathrm{b})}$ and Fredrik Gran \\ Research Group, GN ReSound A/S, Lautrupbjerg 9, 2750 Ballerup, Denmark \\ Finn Jacobsen and Finn Agerkvist \\ Department of Electrical Engineering, Acoustic Technology, Technical University of Denmark, Building 352, \\ 2800 Kgs. Lyngby, Denmark
}

(Received 6 May 2009; revised 16 September 2009; accepted 13 December 2009)

\begin{abstract}
Feedback whistling is one of the severe problems with hearing aids, especially in dynamic situations when the users hug, pick up a telephone, etc. This paper investigates the properties of the dynamic feedback paths of digital hearing aids and proposes a model based on a reflection assumption. The model is compared with two existing models: a direct model and an initialization model, using the measured dynamic feedback paths. The comparison shows that the proposed approach is able to model the dynamic feedback paths more efficiently and accurately in terms of mean-square error and maximum stable gain. The method is also extended to dual-microphone hearing aids to assess the possibility of relating the two dynamic feedback paths through the reflection model. However, it is found that in a complicated acoustic environment, the relation between the two feedback paths can be very intricate and difficult to exploit to yield better modeling of the dynamic feedback paths. (C) 2010 Acoustical Society of America. [DOI: 10.1121/1.3290989]
\end{abstract}

PACS number(s): 43.66.Ts, 43.20.El, 43.60.Ac, 43.60.Uv [AJZ] Pages: 1458-1468

\section{INTRODUCTION}

Feedback is a severe problem in hearing aids, which limits the maximum gain that can be achieved. ${ }^{1}$ The acoustic feedback path is the most significant contributor to the feedback signal although electrical and mechanical paths also exist. $^{2}$ A widely adopted approach to acoustic feedback suppression is feedback cancellation, where the acoustic feedback signal is estimated and then subtracted from the input signal to remove feedback. ${ }^{3}$ The maximum stable gain (MSG) that can be obtained by feedback cancellation depends on how accurately the feedback path is estimated. A perfect match between the modeled and the real feedback path will cancel the feedback signal completely, and the system will be stable for any amount of amplification. ${ }^{4}$

A typical acoustic feedback path in the hearing aids includes the effects of the hearing-aid amplifier, receiver, microphone, the acoustics of the vent or leak, as well as the external acoustics. Except for the fitting and external acoustics, all the parts are nearly constant or very slowly varying with a time frame of weeks or years ${ }^{5}$ when the user has put on the hearing aid. Assume that the user stays still in a stationary acoustic environment, and the feedback path will be almost static. This feedback path is referred to as a "static feedback path." However, in practice, the feedback path is usually subject to dynamic changes. The causes of these changes fall into two categories: (a) changes in the hearing-

\footnotetext{
a) Portions of this work were presented at the 34th IEEE International Conference on Acoustics, Speech and Signal Processing (ICASSP) in April 2009.

${ }^{b}$ Also at Acoustic Technology, Department of Electrical Engineering, Technical University of Denmark. Author to whom correspondence should be addressed. Electronic mail: gm@elektro.dtu.dk
}

aid fitting, including jaw movement, smile, yawn, and cerumen; (b) changes in the external acoustics, including the factors such as room reverberation, a hand, or telephone handset near the ear. The variant feedback path due to these changes is called "dynamic feedback path." In this paper, only the dynamic feedback path for a specific position along the changing course is studied. The temporal characteristics, such as the changing rate at that position, are not addressed.

The properties of feedback paths have been investigated intensively in previous studies, ${ }^{5,2,6}$ where the effect of vent size, the variability of feedback due to smiling, and handset proximity, were discussed. A common observation in these studies is that the largest variation in the acoustic feedback path was found when a hand or telephone handset was placed near the ear. For behind-the-ear (BTE) devices, the maximum variation can go up to $20 \mathrm{~dB}$, whereas for in-theear (ITE) device, it can even go up to $27 \mathrm{~dB}$ in the $2-5 \mathrm{kHz}$ frequency range compared with the static feedback path. ${ }^{6}$

The modeling of the feedback path has also been discussed intensively. However, the majority of the previous work has focused on the static feedback path, trying to model each static component, such as cavities and transducers, by an equivalent electroacoustic model. ${ }^{7-9}$ For dynamic feedback path modeling, there is not very much research up to the present. One study that is relevant measured the variability of feedback path under various conditions and introduced an uncertainty bound model to calculate the robust stability condition for the hearing-aid system. ${ }^{2}$ It provided knowledge about one aspect of the dynamic feedback path properties, i.e., the extent of variability, but did not discuss other acoustic properties and did not investigate the modeling issue either. Another relevant study dealt with feedback cancellation 
in room reverberation. ${ }^{10}$ The focus of that study was the performance of feedback cancellation instead of the properties of the feedback path and its modeling in reverberation.

To track the dynamic aspect of feedback paths in the real world, feedback cancellation in many hearing aids today includes an adaptive feedback path model, where a simple adaptive finite-impulse-response (FIR) filter is used instead of an infinite-impulse-response (IIR) filter, since adaptive IIR filter suffers from the problem of computational complexity, instability, and local minima. ${ }^{11}$ Thus, the estimate of dynamic feedback path is essentially a procedure of updating the coefficients of the adaptive FIR filter.

In general, two models with different ways of using the adaptive FIR filter have been proposed to model the dynamic feedback paths. In the first model, such as the systems proposed in some previous studies, ${ }^{12,13}$ the overall feedback path is represented with the FIR filter. This model is referred to as the "direct model." The second model, such as the systems proposed in other studies, 3,14 incorporates a fixed model and an adaptive FIR filter. The fixed model represents the slowly varying portion of the feedback path (microphone, amplifier, and receiver), whereas the adaptive filter represents the rapidly varying portion (mainly the change of fitting and external acoustics). The fixed model can be either captured by an initialization, ${ }^{3}$ referred to as an "initialization model" in this paper, or roughly approximated as a high-pass filter. ${ }^{14}$ Compared with the direct model, the initialization model generally needs a shorter FIR filter and is computationally more efficient but requires an additional procedure in the hearingaid fitting.

No matter which model is used, effective feedback cancellation requires that the FIR filter should adapt fast enough given the variability of the feedback paths and should be long enough to model all of the salient features. ${ }^{10}$ The feedback path varies at different rates under different conditions. For example, jaw movements will produce changes with a time frame of seconds/minutes. ${ }^{15}$ The picking up of a telephone handset changes the feedback path with a time frame of milliseconds/seconds. Production and disposal of cerumen will result in changes with a time frame of weeks and months, while aging causes changes with a time frame of years. ${ }^{5}$ Usually the adaptation speed is not a problem even in the extreme cases as long as a proper adaptation scheme, for example, frequency-domain adaptation, is used. However, in practice, to maintain sound quality and minimize the artifacts resulted from adaptation errors when a spectrally colored signal is inputted, ${ }^{13}$ the adaptation usually has to slow down and is not fast enough in some occasions. These adaptation problems, although prominent and complicated in the feedback cancellation system, are more related to the on-line adaptation scheme and the characteristics of the input signals, instead of the models of the feedback paths. The focus of this paper is on the modeling. Therefore, these adaptation issues are not addressed in this paper.

As for the sufficient length of the FIR filter in the model, the natural questions are as follows: how many orders of the FIR filter would be "enough" in various dynamic situations, and is there any other model that is more effective in modeling the dynamic feedback paths? In order to address these issues, the characteristics of the dynamic feedback path in addition to variability should be examined. This paper investigates these properties for the most adverse proximity situation that happens frequently and challenges the feedback cancellation most. This paper also describes a novel reflection based model (proposed in a previous publication ${ }^{16}$ ) in detail and extends the previous work thoroughly. The basic idea of the reflection model is to assume that the dynamic feedback path consists of multiple propagation paths and estimate the delays and gains associated with these paths directly. It differs from the two existing models in that the reflection model has an underlying physical assumption, and is more generalized by allowing fractional delays in the model, which will be described in more detail in Sec. II. The effectiveness of the new model is compared with the existing two models mentioned above, i.e., the direct model and the initialization model, using data from measurements. The comparison shows that the proposed model is superior in terms of mean-square error (MSE) and MSG.

For a hearing aid with dual microphones, due to the short distance between the two microphones (normally less than $2 \mathrm{~cm}$ ), the two feedback paths are similar in most situations. This paper investigates the possibility of relating the two dynamic feedback paths using the initialization model and the reflection model. It is shown that in a complicated acoustic environment, the relation between the two feedback paths can be very intricate and difficult to exploit to yield better modeling of the dynamic feedback paths.

The outline of this paper is as follows: in Sec. II, two traditional models are explained and a new reflection model is proposed. In Sec. III, the measurement configuration and procedure are described. The properties of the dynamic feedback paths are discussed in Sec. IV. In Sec. V, the performance of the proposed model is compared with the existing two models and the possibility of using this model for relating the two dynamic feedback paths of a dual-microphone hearing aid is explored based on the measured data. Concluding remarks and directions for future work are given in Sec. VI.

\section{MODELS FOR THE DYNAMIC FEEDBACK PATH}

The general diagram of adaptive feedback cancellation is depicted in Fig. 1. It should be noted that the impulse responses of the microphone and receiver have been included in the impulse response of the feedback path $b(n)$. Although there are various feedback models as mentioned in Sec. I, their estimation of $b(n)$ can all be represented in a compact form $\hat{b}(n, \theta)$, where $n$ denotes the discrete-time index and $\theta$ is a particular parameter set of a model. For example, when the feedback path is modeled by a FIR filter, $\theta$ represents the FIR coefficients. The principle of feedback cancellation is to adjust the parameters $\theta$ in the feedback model so that the modeled feedback path $\hat{b}(n, \theta)$ approximates the true feedback path $b(n)$. The output $v(n)$ is the instantaneous estimation of the feedback signal $f(n)$ and is subtracted from the input signal $s(n)$ to remove the feedback.

In principle, the impulse response $b(n)$ has infinite duration. However, the amplitude of $b(n)$ decays very fast, as 


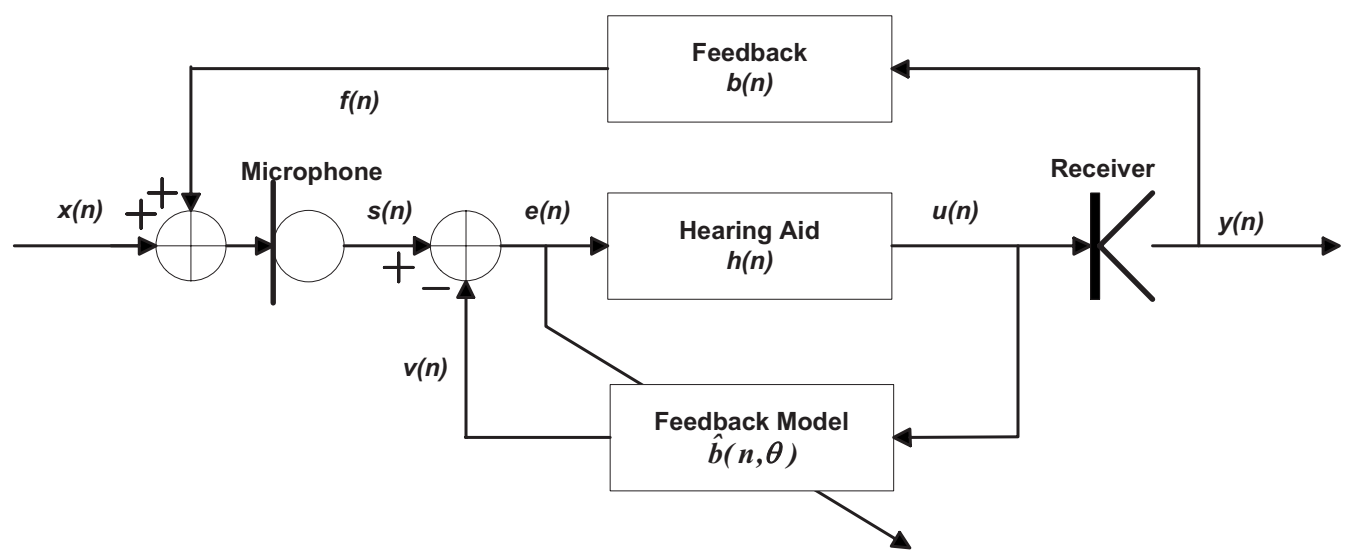

FIG. 1. General diagram of adaptive feedback cancellation. The input to the hearing-aid processing is $s(n)$, which is the sum of desired input signal $x(n)$ and the feedback signal $f(n)$. The processed hearing-aid signal is $u(n)$. The signal output into the ear canal is $y(n)$. The impulse response of the feedback path is $b(n)$, and $v(n)$ is the estimate of $f(n)$ from the modeled feedback path $\hat{b}(n, \theta)$.

shown later in Fig. 5. It is assumed in the following that both $b(n)$ and $\hat{b}(n, \theta)$ are truncated to a sufficient length so that the energy loss in the impulse response due to the truncation is at least $35 \mathrm{~dB}$ below the total energy of $b(n)$ or $\hat{b}(n, \theta)$.

To investigate each feedback model's capability in capturing the true feedback path, the parameters $\theta$ for each model is optimized by minimizing the difference between the modeled feedback $\hat{b}(n, \theta)$ and the actual feedback path. This minimization formulated in the frequency domain is as follows:

$$
\begin{aligned}
& \theta_{\text {opt }}=\arg \min _{\theta}\left\|\mathbf{F}^{H}(\hat{\mathbf{b}}(\theta)-\mathbf{b})\right\|_{2}^{2}, \\
& \mathbf{b}=[b(0), \ldots, b(L-1)]^{T}, \\
& \hat{\mathbf{b}}(\theta)=[\hat{b}(0, \theta), \ldots, \hat{b}(L-1, \theta)]^{T}, \\
& \mathbf{F}=\left[\mathbf{f}_{0}, \mathbf{f}_{1}, \ldots, \mathbf{f}_{L-1}\right] \\
& \mathbf{f}_{k}=\left[1, e^{j \omega_{k}}, \ldots, e^{j \omega_{k}(L-1)}\right]^{T},
\end{aligned}
$$

where \|\|$_{2}$ denotes the Euclidean norm, $L$ is the length of the truncated impulse responses in samples, $\omega_{k}=2 \pi k / L, k$ $=0,1, \ldots, L-1, \mathbf{F}$ is the discrete Fourier transform matrix, and the superscripts $T$ and $H$ denote the transpose and conjugate transpose of a matrix or vector, respectively.

Based on the framework above, Secs. II A-II C will detail each feedback path model by specifying its parameter structure $\theta$, the corresponding optimization procedure, and the analytic optimal solution if it exists. The optimal solution, either in analytic or in numerical form, represents the capability of each feedback model to represent the actual feedback path $b(n)$.

\section{A. Existing models for dynamic feedback paths}

The existing two models for dynamic feedback path are the direct model and the initialization model, both of which can be formulated as a cascade of prefiltering and an adaptive FIR filter.
Let $b_{0}(n)$ and $w(n)$ denote the impulse response of the prefiltering and the adaptive filter, respectively. The modeled feedback $\hat{b}(n, \theta)$ is then the convolution of $b_{0}(n)$ and $w(n)$, i.e.,

$$
\hat{b}(n, \theta)=w(n) \odot b_{0}(n)=\sum_{l=0}^{M-1} w(l) b_{0}(n-l),
$$

where $M$ is the order of $w(n)$, and $\odot$ is the convolution operator.

\section{Direct model}

The feedback path $b(n)$ is always associated with a leading delay, which is the sum of the processing delay in $h(n)$ and the acoustic delay in the transmission path. In the "direct model," the FIR filter $w(n)$ is used to model the "active" portion of the feedback path, whereas the prefiltering $b_{0}(n)$ is simply a delay of $D$ samples introduced to match the leading delay in $b(n)$ and provide a more efficient use of the limited number of taps in $w(n)$. The parameters are therefore of the form

$$
\begin{aligned}
& b_{0}(n)= \begin{cases}1, & n=D \\
0, & \text { otherwise, }\end{cases} \\
& \theta=\{w(n), D\} .
\end{aligned}
$$

The optimal parameters $w_{\text {opt }}(n)$ and $D_{\text {opt }}$ can be obtained by solving the optimization problem in Eqs. (1)-(8).

If the delay $D$ is fixed, the least-squares solution is straightforward:

$$
\mathbf{w}_{\mathrm{opt}}=[b(D), \ldots, b(D+M-1)]^{T} .
$$

If $D$ is not fixed, an easy procedure to obtain the optimal solution is to search for an integer delay $D$ that minimize the distance between $\hat{b}(n, \theta)$ and $b(n)$. 


\section{Initialization model}

To model the feedback path accurately, the direct model in Sec. II A 1 usually needs a very high-order adaptive FIR filter $w(n)$ to cover the active range of the feedback path. One way to improve the modeling efficiency is to use the initialization model.

The feedback path $b(n)$ consists of slowly varying portions such as the responses of microphone, amplifier, receiver, etc. The idea of the initialization model is to use a fixed model, which is initialized during an additional off-line initialization procedure in the hearing-aid fitting, to model these slowly varying portions, and use a short adaptive FIR filter to represent the rapidly varying portions (mainly the change in fitting and external acoustics).

In such an initialization procedure, a "nominal feedback path" is first measured, which is defined as the feedback path without any external reflectors or enclosures near the hearing aid. When the hearing aid is put into use in daily life, the adaptive filter $w(n)$ only needs to model the part that is different from the nominal initialization to capture the timevarying dynamic feedback path. Since the impulse responses of microphone, receiver, etc., are nearly invariant from the initialization to dynamic situations, this different part is mainly resulted from the change in the hearing-aid fitting and external acoustic environments, ${ }^{2}$ which can be modeled by an adaptive filter with a lower order.

In the initialization model, the prefiltering $b_{0}(n)$ in Eq. (6) is a fixed model to represent the impulse response of the nominal feedback path obtained in the initialization. In practice, it can be realized by an autoregressive moving average model (ARMA) model. ${ }^{3}$ To avoid the complicated issue of how to model the initialized feedback path with an ARMA model to get the best generalized performance in feedback cancellation, ${ }^{3,17}$ it is simply assumed that $b_{0}(n)$ is exactly the impulse response of the nominal feedback path. Therefore, the parameter for initialization model is of the form

$$
\theta=\{w(n)\} .
$$

Assume that the filter length of $w(n)$ is $M$, and the impulse response $b_{0}(n)$ is truncated to $L-M+1$ samples and padded with $M-1$ zeros so that the length of $\hat{b}(n, \theta)$, which is the convolution of $w(n)$ and $b_{0}(n)$, equals $L$. The impulse response of the dynamic feedback path $b(n)$ is also assumed to be of length $L$.

The optimal parameter $w_{\text {opt }}(n)$ for real valued $w(n)$ with length $M$ in the initialization model can be found by solving the least-squares problem with Eqs. (1)-(6) and (10):

$$
\begin{aligned}
& \mathbf{w}_{\mathrm{opt}}=\left(\operatorname{diag}\left(\mathbf{F}^{H} \widetilde{\mathbf{b}}_{0}\right) \widetilde{\mathbf{F}}^{H}\right)^{+}\left(\mathbf{F}^{H} \mathbf{b}\right), \\
& \tilde{\mathbf{b}}_{0}=\left[\mathbf{b}_{0}^{T}, \mathbf{0}_{1 \times(M-1)}\right]^{T}, \\
& \mathbf{b}_{0}=\left[b_{0}(0), \ldots, b_{0}(L-M)\right]^{T}, \\
& \widetilde{\mathbf{F}}=\left[\widetilde{\mathbf{f}}_{0}, \widetilde{\mathbf{f}}_{1}, \ldots, \widetilde{\mathbf{f}}_{L-1}\right],
\end{aligned}
$$

$$
\tilde{\mathbf{f}}_{k}=\left[1, e^{j \omega_{k}}, \ldots, e^{j \omega_{k}(M-1)}\right]^{T},
$$

where $\omega_{k}=2 \pi k / L, k=0,1, \ldots, L-1, \operatorname{diag}(\cdot)$ forms a diagonal matrix with diagonal elements specified in $(\cdot),(\cdot)^{+}$is a pseudoinverse defined as $(\cdot)^{+}=\left((\cdot)^{H}(\cdot)\right)^{-1}(\cdot)^{H}$, and $\mathbf{0}_{1 \times(M-1)}$ represents a zero vector of size $1 \times(M-1)$. The matrix $\widetilde{\mathbf{F}}$ is a partial discrete Fourier transform matrix which results from the fact that $w(n)$ is only of the length $M$.

\section{B. Reflection model for dynamic feedback paths 1. Model formulation}

The initialization model $\hat{b}(n, w(n))$ formulated in Eq. (6) can also be regarded as a weighted sum of the nominal impulse response $b_{0}(n)$ and its delayed replicas with integer delays.

In typical dynamic situations where there is room reverberation or a handset placed close to the hearing aid, the feedback path is a composition of reflection and refraction, where reflection usually dominates. In addition to attenuation or amplification in the amplitude, a certain delay is associated with each reflected component. These physical delays, when transformed into the digital domain, are possibly fractional in terms of samples. Therefore, the model in Eq. (6) is generalized to a new model as follows:

$$
\begin{aligned}
& \hat{b}(n, \theta)=\sum_{l=0}^{M-1} w(l) b_{0}\left(n-d_{l}\right), \\
& \theta=\left\{w(l), d_{l}\right\},
\end{aligned}
$$

where $d_{l}$ is the delay of the $l$ th replica, $d_{l}>d_{l-1} \geq 0$, and $l$ $=1, \ldots, M-1$. Although the delays $d_{l}$ in the model equations (16) and (17) are allowed to be fractional, the actual subsample implementation needs time-domain interpolation or frequency-domain multiplication. The latter is more efficient and is given in Sec. II B 2.

In this model, the impulse response of the nominal feedback path, measured during initialization without significant disturbances such as reflections, is regarded as an approximation of the direct path. The dynamic feedback path is modeled as a sum of reflection components with delay $d_{l}$ and gain $w(l)$. This model is thus named "reflection model." When $d_{l}=l$, the reflection model reduces to the initialization model. Therefore, the reflection model is more general and expected to capture the dynamic feedback path better than the initialization model since it represents more accurately what happens in the physical world.

\section{Delay estimation}

The optimal delays $d_{l, \text { opt }}$ and weights $w_{\text {opt }}(l)$ for the reflection model can be found by solving the optimization problem given by Eqs. (1)-(5), (16), and (17), which is a nonlinear optimization problem.

However, efficient time delay (TDE) and amplitude estimation techniques have been investigated intensively in many fields such as radar, sonar, radio navigation, geophysical/seismic exploration, wireless communication, and medical imaging. The most well-known approach is 
based on the matched filter, ${ }^{18}$ whose resolution capability is unfortunately limited to the reciprocal of the signal bandwidth. For dynamic feedback path modeling, the difference between delays can be very small due to the handset proximity effect. Therefore, TDE techniques with high resolution should be used. Among these techniques, algorithms such as multiple signal classification, linear prediction, and maximum likelihood are not very well suited here since they are best for complex-valued signals with special spectral shapes. ${ }^{19}$ A weighted Fourier transformation and relaxation (WRELAX) based method ${ }^{20}$ is found to be very robust to address the problem. First, the cost function is defined as follows:

$$
C_{1}\left(\left\{w(l), d_{l}\right\}_{l=0}^{M-1}\right)=\left\|\mathbf{B}-\sum_{l=0}^{M-1} w(l)\left[\mathbf{S a}\left(d_{l}\right)\right]\right\|_{2}^{2},
$$

where

$$
\begin{aligned}
& \mathbf{B}=\mathbf{F}^{H} \mathbf{b}, \\
& \mathbf{S}=\operatorname{diag}\left(\mathbf{F}^{H} \tilde{\mathbf{b}}_{0}\right), \\
& \mathbf{a}\left(d_{l}\right)=\left[1, e^{-j(2 \pi / L) d_{l}}, \ldots, e^{-j(2 \pi / L) d_{l}(L-1)}\right]^{T} .
\end{aligned}
$$

Equation (18) is simply a formulation of the inner part of Eq. (1) with Eqs. (16) and (17) inserted. Minimizing the cost function $C_{1}\left(\left\{w(l), d_{l}\right\}_{l=0}^{M-1}\right)$ with respect to $w(l)$ and $d_{l}$ is the problem of interest. To address this optimization problem, denote

$$
\begin{aligned}
& \mathbf{B}_{l}=\mathbf{B}-\sum_{i=0, i \neq l}^{M-1} \hat{w}(i)\left[\mathbf{S a}\left(\hat{d}_{i}\right)\right], \\
& C_{2}\left(w(l), d_{l}\right)=\left\|\mathbf{B}_{l}-w(l) \mathbf{S a}\left(d_{l}\right)\right\|_{2}^{2} .
\end{aligned}
$$

The cost function $C_{2}\left(w(l), d_{l}\right)$ assumes that the other reflection components have been estimated as $\hat{w}(i)$ and $\hat{d}_{i}$, where $i=0,1, \ldots, M-1, i \neq l$, except the $l$ th component, and minimizing this cost function yields the estimates $\hat{w}(l)$ of $w(l)$ and $\hat{d}_{l}$ of $d_{l}$ as $^{20}$

$$
\begin{aligned}
& \hat{d}_{l}=\arg \max _{d_{l}}\left|\mathbf{a}^{H}\left(d_{l}\right)\left(\mathbf{S}^{*} \mathbf{B}_{l}\right)\right|^{2}, \\
& \hat{w}(l)=\left.\frac{\mathbf{a}^{H}\left(d_{l}\right)\left(\mathbf{S}^{*} \mathbf{B}_{l}\right)}{\|\mathbf{S}\|_{2}^{2}}\right|_{d_{l}=\hat{d}_{l}},
\end{aligned}
$$

where the asterisk denotes the complex conjugate. If $w(l)_{l=0}^{M-1}$ are assumed to be real numbers, minimizing $C_{2}\left(w(l), d_{l}\right)$ with respect to $w(l)$ and $d_{l}$ yields ${ }^{20}$

$$
\begin{aligned}
& \hat{d}_{l}=\arg \max _{d_{l}}\left\{\mathfrak{R}\left[\mathbf{a}^{H}\left(d_{l}\right)\left(\mathbf{S}^{*} \mathbf{B}_{l}\right)\right]\right\}^{2}, \\
& \hat{w}(l)=\left.\frac{\Re\left[\mathbf{a}^{H}\left(d_{l}\right)\left(\mathbf{S}^{*} \mathbf{B}_{l}\right)\right]}{\|\mathbf{S}\|_{2}^{2}}\right|_{d_{l}=\hat{d}_{l}},
\end{aligned}
$$

where $\mathfrak{R}[\cdot]$ denotes the real part of $(\cdot)$.
To estimate $\hat{d}_{l}$ using Eq. (26), an initial estimate of $d_{l}$ is first obtained by locating the maximum peak in the magnitude of the $K$-point $(K \geq L)$ fast Fourier transform (FFT) of $\mathbf{S}^{*} \mathbf{B}_{l}$ with padded zeros. This process can also be regarded as a matched filtering in the frequency domain. Then a search for the $\hat{d}_{l}$ that maximizes $\mathfrak{R}\left\{\left[\mathbf{a}^{H}\left(d_{l}\right)\left(\mathbf{S}^{*} \mathbf{B}_{l}\right)\right]\right\}^{2}$ is made in a small range around the initial estimate based on golden section search and parabolic interpolation. ${ }^{21,22}$

The WRELAX estimates the delays $d_{l}$ and amplitudes $w_{l}$ in the frequency domain to avoid fractional delay interpolation. The general idea is to cross-correlate $b(n)$ and $b_{0}(n)$ in the frequency domain to find the coarse delays and gains of the replicas by identifying the peaks of the cross-correlation. Later an iterative search is performed by keeping one replica of $b_{0}(n)$ at a time (removing the other identified replicas from $b(n))$, repeating the cross-correlation and locating the peak to find a better delay and gain estimation for that replica. This process is iterated until the relative change in the cost function in Eq. (18) is below the threshold $\epsilon$. The detailed steps are given below.

Step 1: For $M=1$ obtain $\left\{\hat{w}(l), \hat{d}_{l}\right\}_{l=0}$ from $\mathbf{B}$ by using Eqs. (26) and (27).

Step 2: For $M=2$ compute $\mathbf{B}_{1}$ with Eq. (22) by using $\left\{\hat{w}(l), \hat{d}_{l}\right\}_{l=0}$ obtained in Step 1. Obtain $\left\{\hat{w}(l), \hat{d}_{l}\right\}_{l=1}$ from $\mathbf{B}_{1}$ by using Eqs. (26) and (27). Next recompute $\mathbf{B}_{0}$ by using $\left\{\hat{w}(l), \hat{d}_{l}\right\}_{l=1}$ and redetermine $\left\{\hat{w}(l), \hat{d}_{l}\right\}_{l=0}$ from $\mathbf{B}_{0}$. Iterate these two substeps until the relative change in $C_{1}\left(\left\{w(l), d_{l}\right\}_{l=0}^{M-1}\right)$ between two consecutive iterations is below the threshold $\epsilon$.

Step 3: For $M=3$ compute $\mathbf{B}_{2}$ with Eq. (22) by using $\left\{\hat{w}(l), \hat{d}_{l}\right\}_{l=0}^{1}$ obtained in Step 2. Obtain $\left\{\hat{w}(l), \hat{d}_{l}\right\}_{l=2}$ from $\mathbf{B}_{2}$. Next recompute $\mathbf{B}_{0}$ by using $\left\{\hat{w}(l), \hat{d}_{l}\right\}_{l=1}^{2}$ and redetermine $\left\{\hat{w}(l), \hat{d}_{l}\right\}_{l=0}$ from $\mathbf{B}_{0}$. Then compute $\mathbf{B}_{1}$ by using $\left\{\hat{w}(l), \hat{d}_{l}\right\}_{l=0,2}$ and redetermine $\left\{\hat{w}(l), \hat{d}_{l}\right\}_{l=1}$. Iterate these three substeps until the relative change in $C_{1}\left(\left\{w(l), d_{l}\right\}_{l=0}^{M-1}\right)$ between two consecutive iterations is below the threshold $\epsilon$.

Remaining Steps: Continue similarly until $M$ is equal to the desired number of paths.

In contrast to some estimation algorithms, such as the expectation-maximization algorithm, ${ }^{23}$ the WRELAX is not sensitive to the initial parameters since it uses a matched filter to find these initial values. It is bound to converge to at least a local minimum point, which is at least a better solution than what the traditional matched filter can yield. The convergence speed of WRELAX depends on the time delay spacing of the different reflected paths. ${ }^{20}$

\section{Models for dual-microphone hearing aids}

For hearing aids with dual microphones, the feedback problem involves a dynamic feedback path for the front microphone, denoted as $b_{f}(n)$, and a path for the rear microphone, denoted as $b_{r}(n)$. One way of dealing with the two paths is to model them individually by using one of the three models described above. An alternative approach involves 


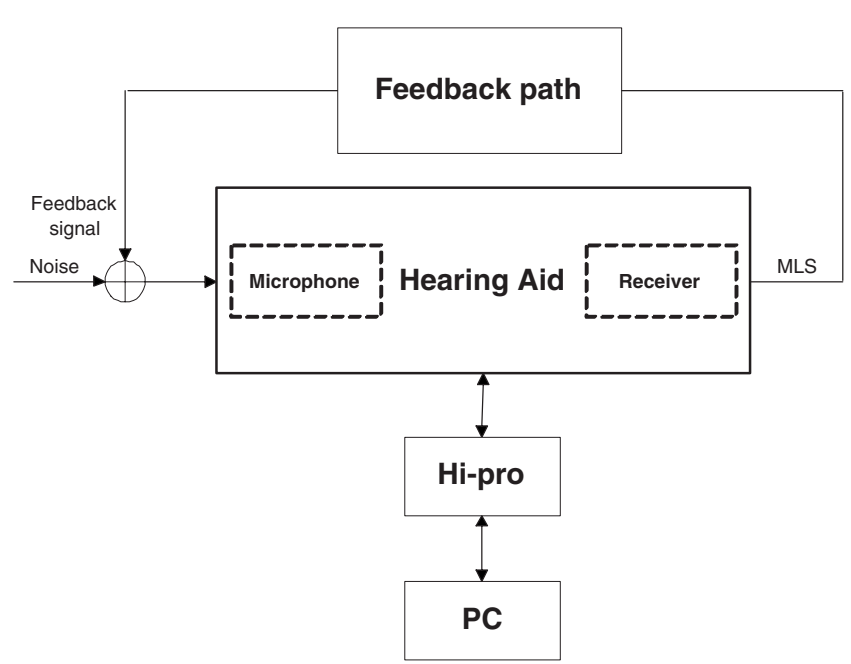

FIG. 2. The block diagram of the measurement system for measuring the feedback paths. The feedback path is illustrated which includes the external acoustics, for example, a Kemar head. The receiver sends out a MLS and the feedback signal is picked up by the microphone with ambient noise together. The data are fed into the computer through HI-PRO.

exploiting the relation between the two similar feedback paths by fitting one dynamic feedback path with the other dynamic path. There are two ways of fitting.

The first approach for the fitting, which is similar to the initialization model [cf. Eq. (6)], is

$$
\hat{b}_{r}(n)=\sum_{l=0}^{M-1} w(l) b_{f}(n-l) \text {. }
$$

The second approach for the fitting, which is similar to the reflection model [cf. Eq. (16)], is

$$
\hat{b}_{r}(n)=\sum_{l=0}^{M-1} w(l) b_{f}\left(n-d_{l}\right)
$$

The estimate of $w_{\text {opt }}(n)$ and/or delays $d_{l, \text { opt }}$ can be found in similar ways described in Secs. II A 2 and II B.

\section{MEASUREMENT OF DYNAMIC FEEDBACK PATHS}

The nominal and dynamic feedback paths have been measured using a commercial open-fitting BTE device with two microphones from GN ReSound A/S. The hearing aid was mounted on the head of Kemar Manikin type 45BA made by G.R.A.S. Sound \& Vibration A/S. ${ }^{24}$

The impulse responses of the feedback paths were measured by sending out a maximum-length sequence (MLS) with a period of 255 samples through the receiver, repeating it 1000 periods, and averaging the responses to get a high signal-to-noise ratio (SNR) for the feedback path response relative to random ambient noise. The sampling frequency was $15625 \mathrm{~Hz}$. Figure 2 illustrates the measurement diagram where a HI-PRO universal programming interface for hearing instruments made by GN Otometrics A/S (Ref. 25) is used to feed the collected data into a computer to calculate the impulse responses. The detailed procedure of the impulse response measurement can also be found in Ref. 10.

The MLS is emitted at around $85 \mathrm{~dB}$ sound pressure level (SPL) and transmitted to the microphone through the

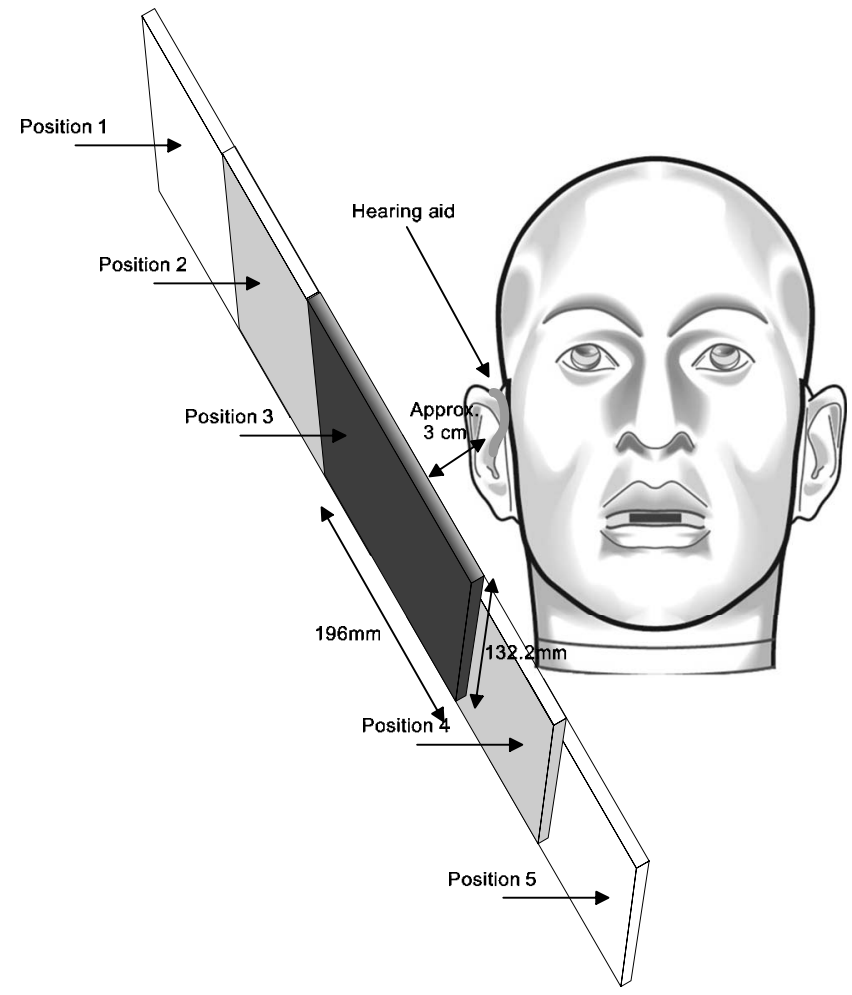

FIG. 3. The measurement setup for dynamic feedback paths with rigid surface. The hearing aid is mounted on the Kemar head. Positions 1 and 2 are symmetrical to positions 4 and 5 with regard to position 3, which is right at the side of the hearing aid.

feedback. The feedback path shapes the signal based on its frequency response. The internal noise in the microphone is less than $28 \mathrm{~dB}$ SPL and the ambient noise is less than $30 \mathrm{~dB}$ SPL. The average over 1000 cycles of MLS increases the overall SNR by $30 \mathrm{~dB}$. This setup has been found to be very reliable. The preliminary test shows that when the measurements are repeated for ten times for a single feedback path, the variance in the measured impulse responses is around 36 $\mathrm{dB}$ below the average response, which is sufficient for feedback path modeling.

The nominal feedback paths for both the front and rear microphones are measured. The measured nominal feedback path for the front microphone is denoted as $b_{f, 0}(n)$ and for the rear microphone $b_{r, 0}(n)$.

Since it was found that the movement of jaw, mouth, etc., had little effect on the feedback path variations, ${ }^{5,2}$ and the adaptive feedback cancellation in hearing aids today usually handles these situations very well in practice, the measurement of dynamic feedback paths focused on the proximity effect.

Seven dynamic feedback paths were measured for both the front and rear microphones. A rigid metal surface of dimension $196 \times 132.6 \mathrm{~mm}^{2}$ was used and faced to the hearing aid mounted on the Kemar head. The surface was moved along the lateral side gradually toward the hearing aid from position 1 to position 3 and later from position 3 to position 5, as shown in Fig. 3. The perpendicular distance between the rigid surface and the hearing aid was kept at around $3 \mathrm{~cm}$ during the movement. The impulse responses of the dynamic feedback path at these five positions measured from the front 

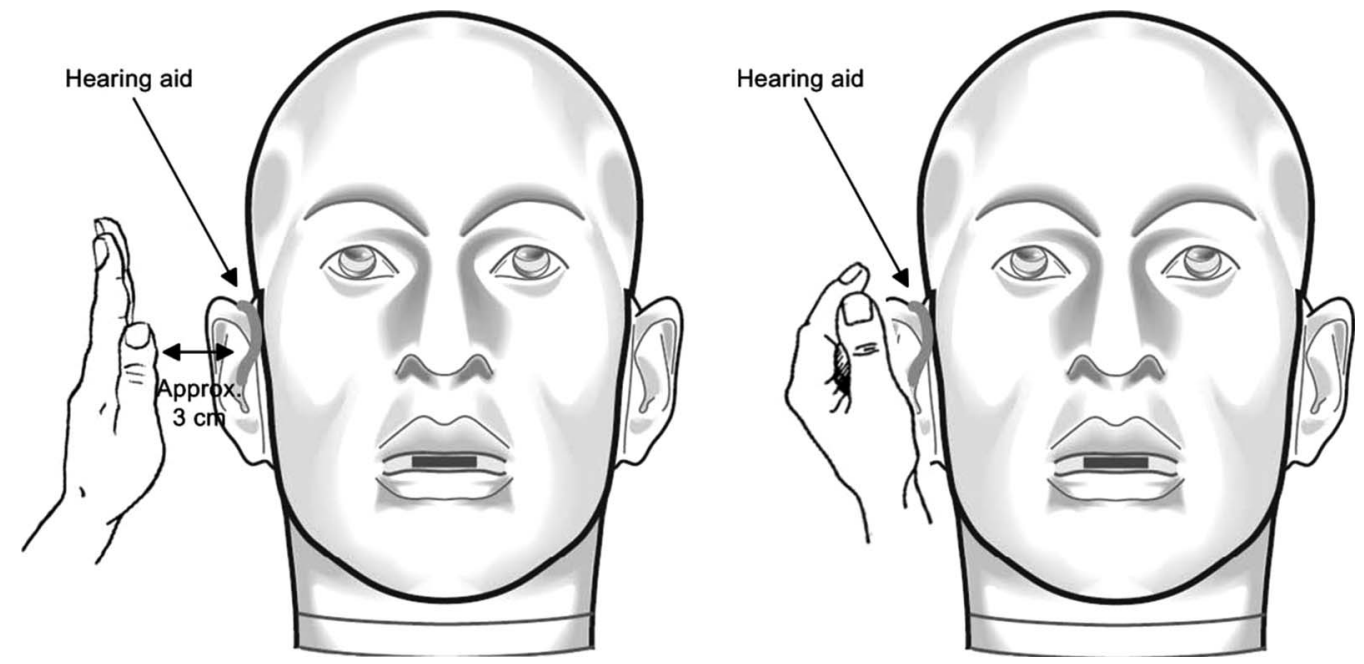

FIG. 4. The measurement setup for dynamic feedback paths with palm. The left figure represents an outstretched palm facing the hearing aid, and the right figure depicts the cupped hand around the hearing aid.

microphone are denoted as $b_{f, 1}(n)-b_{f, 5}(n)$ and from the rear microphone $b_{r, 1}(n)-b_{r, 5}(n)$. Apart from the rigid surface, two additional dynamic feedback paths were measured with an outstretched palm facing the hearing aid on its lateral side at a distance of around $3 \mathrm{~cm}\left[b_{f, 6}(n), b_{r, 6}(n)\right]$ and with a palm wrapping around the hearing aid $\left[b_{f, 7}(n), b_{r, 7}(n)\right]$, as illustrated in Fig. 4.

Therefore, altogether eight impulse responses were measured for both front and rear microphones including the static feedback path (nominal feedback) and seven dynamic feedback paths. All the impulse responses are of the length 255 samples. The rigid surface measurements are artificial tests for reflections whereas the palm measurements mimic the most adverse situations for feedback cancellation in real life.

The frequency responses of these feedback paths are shown in Fig. 5, where only the responses from the front microphone are illustrated since the responses from the rear are similar.
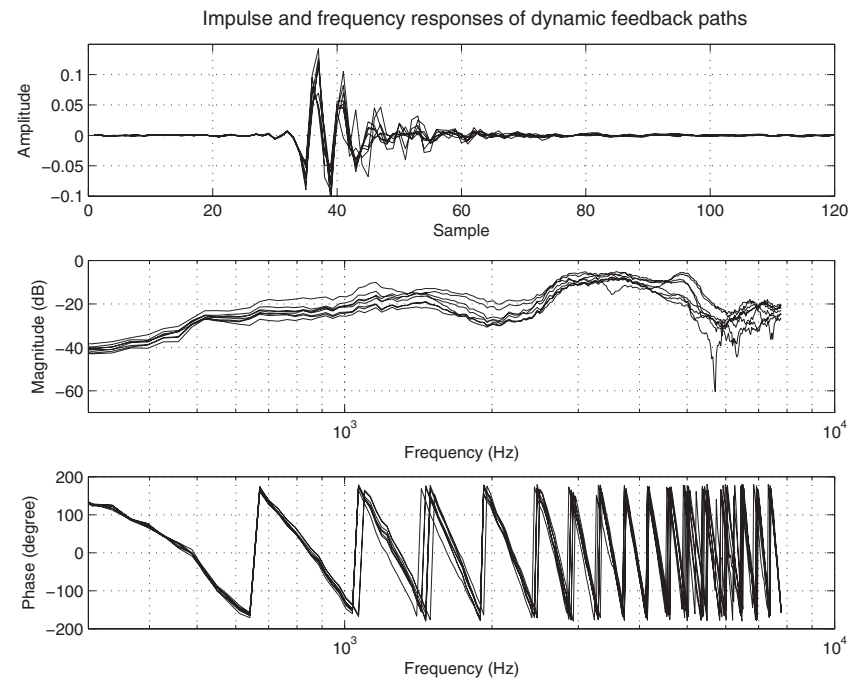

FIG. 5. Impulse responses and frequency responses of measured eight feedback paths (one nominal and seven dynamic feedback paths).

\section{PROPERTIES OF DYNAMIC FEEDBACK PATHS}

In this section, the general characteristics of the measured dynamic feedback paths are investigated first. Later, the reflection model proposed in Sec. II B is used to model these paths and reveal other aspects of the characteristics of dynamic feedback paths.

\section{A. Variability of dynamic feedback paths}

The sample mean and sample estimate of the standard deviation of the measured dynamic feedback paths have been calculated as a function of frequency. The mean and standard deviation do not reveal all the properties of the dynamic feedback paths but give an indication of the dynamic range of the data. If it can be assumed that the variation in spectrum magnitude for each frequency is normally distributed, then the $95 \%$ confidence interval of the expected value for seven measurements is $\left[\overline{\mathbf{B}}_{f}-0.925 \mathbf{S}_{f}, \overline{\mathbf{B}}_{f}+0.925 \mathbf{S}_{f}\right]$ and $\left[\overline{\mathbf{B}}_{r}\right.$ $\left.-0.925 \mathbf{S}_{r}, \overline{\mathbf{B}}_{r}+0.925 \mathbf{S}_{r}\right]$ for the front- and rear-microphone dynamic paths, respectively, where $\overline{\mathbf{B}}_{f}$ and $\overline{\mathbf{B}}_{r}$ are sample means, and $\mathbf{S}_{f}$ and $\mathbf{S}_{r}$ are sample estimates of the standard deviation.

They are calculated as follows:

$$
\begin{aligned}
\overline{\mathbf{B}}_{f} & =\frac{1}{7} \sum_{l=1}^{7}\left|\mathbf{F}^{H} \mathbf{b}_{f, l}\right|, \\
\mathbf{S}_{f} & =\sqrt{\frac{1}{7-1} \sum_{l=1}^{7}\left(\left|\mathbf{F}^{H} \mathbf{b}_{f, l}\right|-\overline{\mathbf{B}}_{f}\right)^{2}},
\end{aligned}
$$

where

$$
\mathbf{b}_{f, l}=\left[b_{f, l}(0), \ldots, b_{f, l}(L-1)\right]^{T} .
$$

Note that $\overline{\mathbf{B}}_{f}$ and $\mathbf{S}_{f}$ are both vectors. The symbol $|\cdot|$ in Eq. (30) and (.) $)^{2}$ in Eq. (31) denote the elementwise absolute operation and elementwise square of the vector in $(\cdot)$, respectively. The $\overline{\mathbf{B}}_{r}$ and $\mathbf{S}_{r}$ can be calculated similarly. 


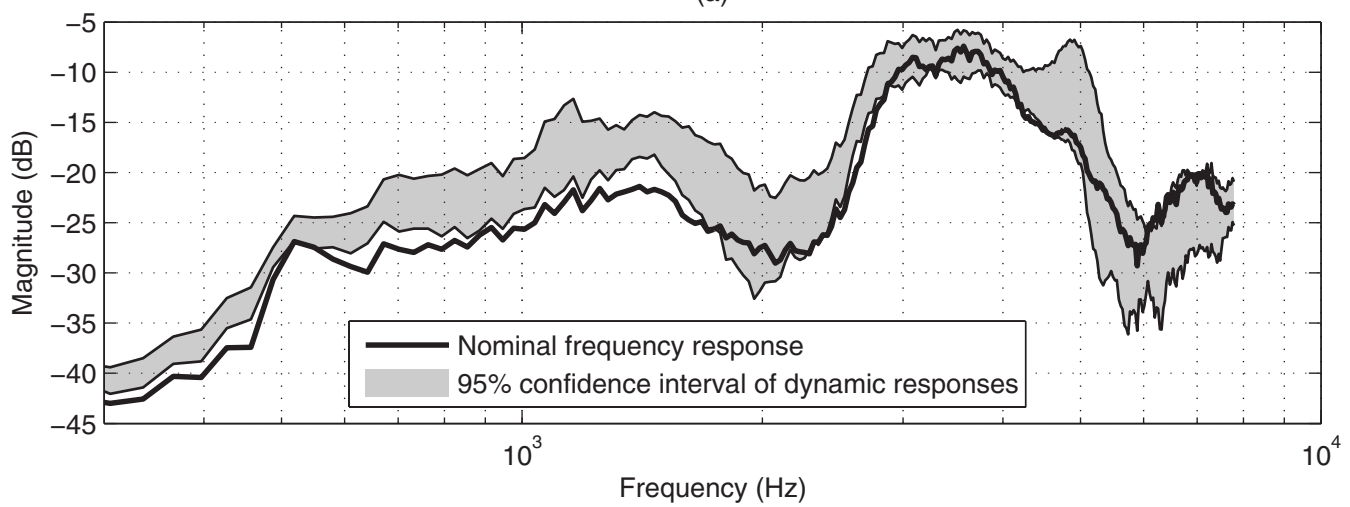

(b)

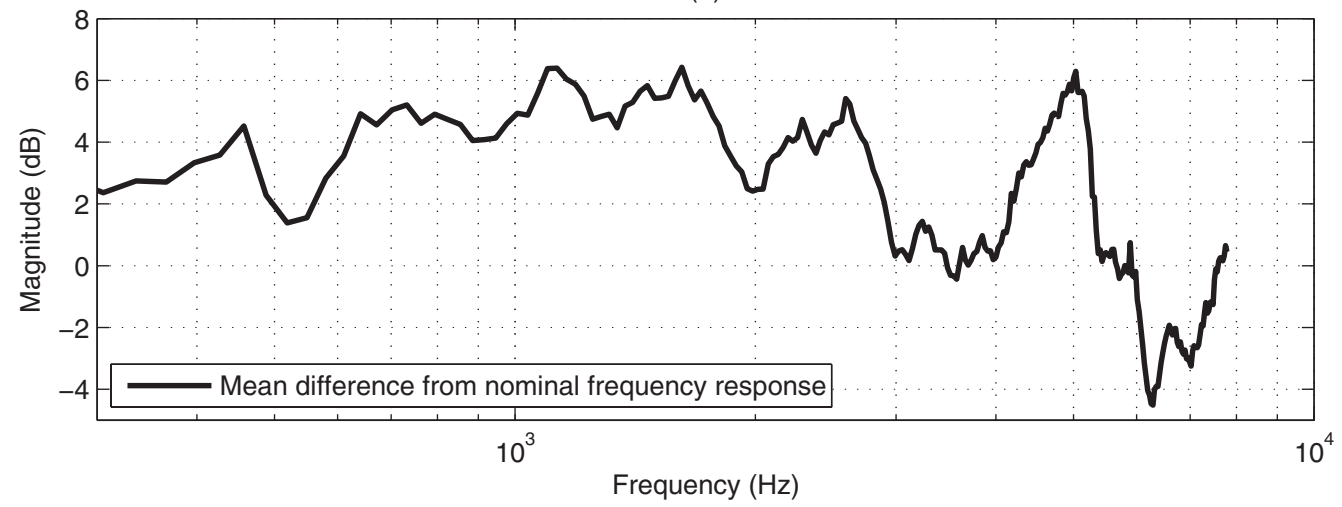

FIG. 6. Variability of dynamic feedback paths: (a) shows the nominal frequency response and a shaded region representing the $95 \%$ confidence interval of the expected frequency response of dynamic feedback paths; (b) illustrates the mean difference between the responses of dynamic feedback paths and the nominal feedback path.

The variability results are shown in Fig. 6(a). The shaded region represents the uncertainty in the expected magnitude of frequency responses of the dynamic feedback paths. It should be noted that the variability illustrated here is obtained with one single head and does not include the intersubject variability. The general trend is that at low frequencies, the uncertainty is small, but above $1 \mathrm{kHz}$, the uncertainty increases and goes up to $10 \mathrm{~dB}$ at some frequencies. The magnitude curve of the frequency response of the nominal feedback path lies below this shaded region at frequencies lower than $1750 \mathrm{~Hz}$, which means that the rigid surface or hand in the measurements generally boosted the feedback responses at low frequencies. Above $1750 \mathrm{~Hz}$, most of the nominal curve lies within the shaded region, and above 5 $\mathrm{kHz}$, nearly all of the curve lies inside the region. This indicates that the change in feedback path at high frequencies is not a general boost but resulted from complicated effects such as peaks and valleys. This trend agrees with the results from other studies, ${ }^{5,2,6}$ despite the minor differences in the measured feedback paths due to different devices, test environments, etc.

The difference in $\mathrm{dB}$ between the sample mean of the magnitude of dynamic feedback paths $\overline{\mathbf{B}}_{f}$ and the magnitude of the nominal feedback path is illustrated in Fig. 6(b), which essentially removes the effects of microphone, receiver, etc. A dominant peak was found at $5035 \mathrm{~Hz}$. This can be interpreted as a strong reflection from an object placed at a distance of $1 / 5035 \times 343 / 2 \mathrm{~m} \approx 0.034 \mathrm{~m}$, where $343 \mathrm{~m} / \mathrm{s}$ is the speed of sound at $20{ }^{\circ} \mathrm{C}$ and the factor of 2 is used to calculate a single-way transmission length. This is approximately the distance between the hand/rigid surface and the hearing aid in the measurement.

\section{B. Reflections in dynamic feedback paths}

The reflection phenomenon in dynamic feedback paths with a telephone handset near the hearing aid has been pointed out in several previous papers. ${ }^{3,5,6}$ It features peaks or other complicated effects in the high frequency range of the measured feedback responses. The dominant peak in the Fig. 6(b) also indicates the occurrence of a reflection.

However, physically, there could be many other reflections besides the dominant one, especially when the object is placed very close to the hearing aid. The reflection model proposed in this paper can be used to estimate these reflections.

In WRELAX of the reflection model, one zero is padded to all of the impulse responses of the measured feedback paths so that the truncated length $L$ becomes 256 . The number of points in the FFT for initial delay estimation, $K$, is set to 4096 to get a good initial guess, the search region around the initial estimate $\hat{d}_{l, \text { ini }}$ in samples is set to be $\left[\hat{d}_{l, \text { ini }}\right.$ $\left.-\frac{1}{32}, \hat{d}_{l, \text { ini }}+\frac{1}{32}\right]$, and the threshold for the stopping criterion $\epsilon$ is set as $10^{-4}$.

The reflection model estimates the $w(l)$ and $d_{l}$, where $l$ $=0,1, \ldots, M-1$, for all the measured data. In Figs. 

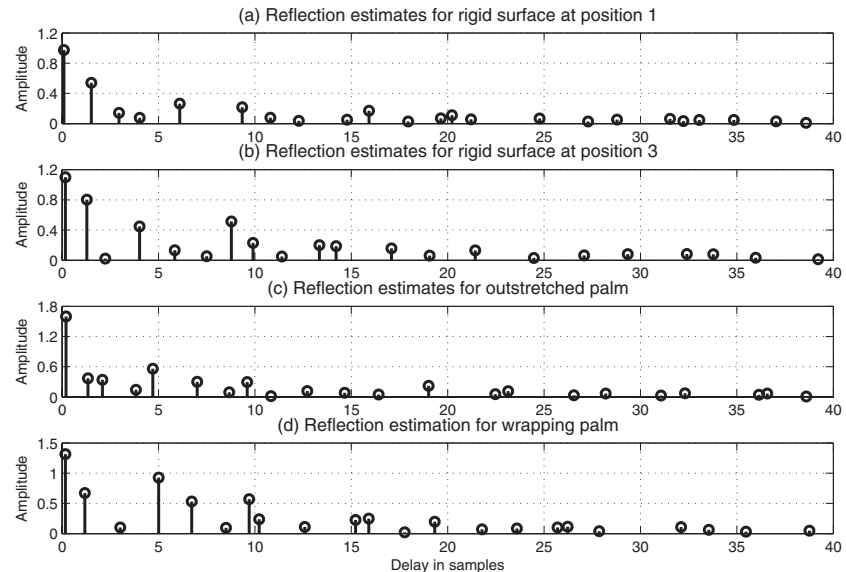

(e) MSE as a function of model order

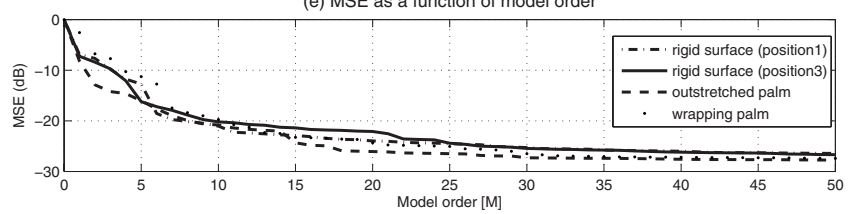

FIG. 7. (a)-(d) illustrate the reflection estimates in terms of delay in samples $\hat{d}_{l}$ and amplitude $\hat{w}(l)$ based on the measured data for rigid surface at positions 1 and 3, outstretched palm, and wrapping palm; (e) shows how the MSE, i.e., $C_{1}\left(\left\{w(l), d_{l}\right\}_{l=0}^{M-1}\right)$ decreases as the number of reflection components $M$ increases.

$7(\mathrm{a})-7(\mathrm{~d})$, the results for the rigid surface at positions 1 and 3 , and palm outstretched and wrapped around the hearing aid, are illustrated. The MSE of the estimated feedback path, i.e., $C_{1}\left(\left\{w(l), d_{l}\right\}_{l=0}^{M-1}\right)$ defined in Eq. (18), is given in Fig. 7(e) as a function of the model order. As can be seen from the figure, in all the four cases, the highest peak is located near 0 sample delay, which implies that the assumption that the nominal feedback path is approximately the direct path is valid. The reason why the amplitude is not exactly unity and the delay is not exactly 0 is that the nominal feedback path itself consists of a number of small reflections due to the presence of pinna and head.

As the delay increases, the estimated amplitude generally decays, which complies with the physical law that longer transmission path results in larger attenuation (transmission loss). ${ }^{26}$ Almost all of the significant peaks in the four cases happen before a delay of ten samples, corresponding to a single-way transmission length of $0.106 \mathrm{~m}$, which is a reasonable range for late reflection paths, e.g., a path consisting of multiple reflections between reflecting objects, the ear, and the hearing aid before it reaches the microphone.

There are fewer significant reflections (with amplitude larger than 0.4) in the cases of the rigid surface at position 1 and outstretched palm than in the cases of the rigid surface at position 3 and wrapping hand. This agrees with the fact that the reflections in the latter two cases are more complicated.

The case with the wrapping hand has the most irregular reflection pattern, in which large reflections are distributed widely in the delay range from zero to ten samples. The reflections for this situation after a delay of ten samples are generally as small as in the other cases. This shows that the wrapping hand introduces large reflections with short delays.

In the experiments with the rigid surface and outstretched palm, the distance was kept at around $3 \mathrm{~cm}$, corre-
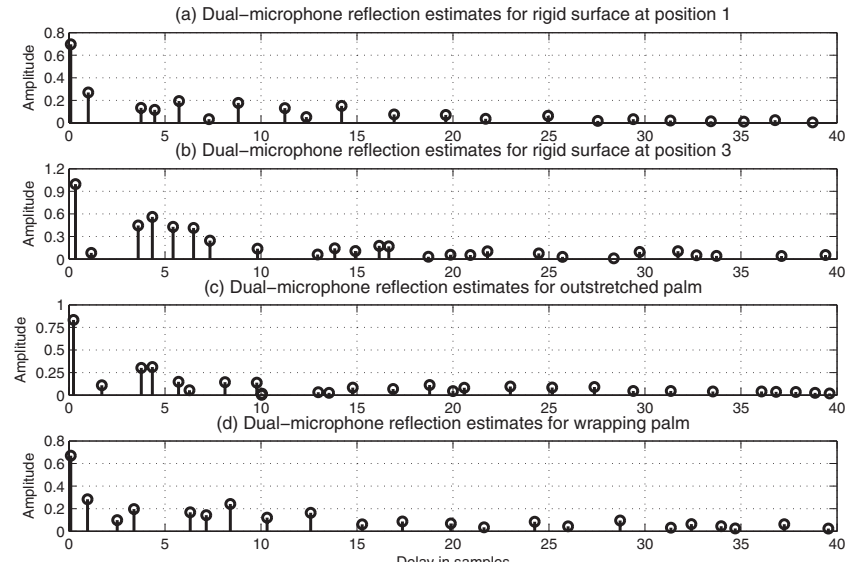

(e) MSE as a function of model order

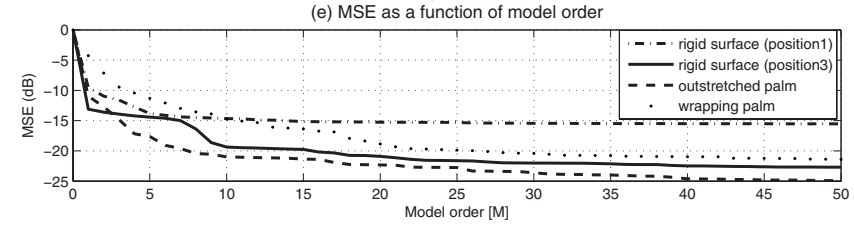

FIG. 8. (a)-(d) illustrate the reflection estimates for the dual-microphone BTE hearing aid assuming that the rear response is a sum of reflected replicas of the front response in terms of delay in samples $\hat{d}_{l}$ and amplitude $\hat{w}(l)$ based on the measured data for rigid surface at positions 1 and 3 , outstretched palm, and wrapping palm; (e) shows how the MSE, i.e., $C_{1}\left(\left\{w(l), d_{l}\right\}_{L=0}^{M-1}\right)$ decreases as the number of reflection components $M$ increases.

sponding to a delay of 2.73 samples and a peak at $5717 \mathrm{~Hz}$ in the magnitude response. The peak has been verified in Sec. IV A, whereas for all the cases in Figs. 7(a)-7(d), the peak does not seem to be very dominant, although there is a reflection component located at around 2.73 samples. A possible reason is that the dominant reflections were influenced by the presence of the pinna and the head, depending on how the reflecting object was positioned. However, this influence is different for different measurements. Therefore, it is averaged out and the dominant peak shows up at a correct location in the average magnitude response [see Fig. 6(b) at 5035 $\mathrm{Hz}]$.

For the dual-microphone situation, the estimation results are shown in Fig. 8. For each case, the response of the feedback path at the rear microphone is fitted by that at the front microphone using the proposed reflection model. As seen from Fig. 8(e), the MSE converges at a relatively higher level compared with Fig. 7(e), especially for the case where the rigid surface is placed at position 1 . Therefore, the usage of a reflection model to relate the two feedback paths of a dual-microphone BTE hearing aid is not very effective.

\section{PERFORMANCE OF THE PROPOSED MODEL}

In this section, the proposed reflection model is compared with the two existing model in terms of MSE and MSG. The performance of the reflection model for dualmicrophone situation is also investigated.

\section{A. Performance metric of feedback path models}

To evaluate the performance of a feedback model, two metrics are usually used: MSE and MSG. The MSE de- 
scribes the average distance between the modeled and the real feedback paths. The MSG indicates the maximum gain without instability assuming a flat frequency response in the hearing-aid processing and the worst case for the phase. The MSG is determined by the frequency at which the mismatch between the feedback model and the actual feedback path is the largest. ${ }^{10}$ Assume that all the models are optimized, the resultant MSE and MSG are defined as $\mathrm{MSE}_{c}$ and $\mathrm{MSG}_{c}$, respectively,

$$
\begin{aligned}
\operatorname{MSE}_{c} & =20 \log _{10}\left(\frac{\left\|\mathbf{F}^{H} \hat{\mathbf{b}}\left(\theta_{o p t}\right)-\mathbf{b}\right\|_{2}^{2}}{\left\|\mathbf{F}^{H} \mathbf{b}\right\|_{2}^{2}}\right), \\
\mathrm{MSG}_{c} & =20 \log _{10}\left(\min _{k} \frac{1}{\left|\mathbf{f}_{k}^{H}\left(\hat{\mathbf{b}}\left(\theta_{o p t}\right)-\mathbf{b}\right)\right|}\right) .
\end{aligned}
$$

With a specific model and parameters $\theta$, the $\mathrm{MSE}_{c}$ and $\mathrm{MSG}_{c}$ are the lowest achievable MSE and the highest achievable MSG, respectively. $\mathrm{MSE}_{c}$ and $\mathrm{MSG}_{c}$ are, in fact, limited by the amount of undermodeled feedback path, the residual feedback path that cannot be modeled due to the limited number of degrees of freedom in the parameter $\theta$ and/or the lack of flexibility in the model form. A more descriptive model with larger degrees of freedom in the parameters $\theta$ will yield less undermodeling, lower $\mathrm{MSE}_{c}$ and larger $\mathrm{MSG}_{c}$.

\section{B. Results}

For each measured dynamic feedback path, the parameters in the models were first optimized to calculate the $\mathrm{MSE}_{c}$ and $\mathrm{MSG}_{c}$. The filter length $M$ was varied from 1 to 50. To achieve the best performance, the delay $D$ was not fixed in the direct model.

It is found that for all the seven dynamic paths and all the values $M$, the reflection model outperforms the initialization model and the direct model in terms of $\mathrm{MSE}_{c}$ and $\mathrm{MSG}_{c}$. The direct model performs worst in all the cases. To demonstrate the performance of each model in dynamic situations, $\mathrm{MSE}_{c}$ and $\mathrm{MSG}_{c}$ are averaged over the seven dynamic paths. The results are illustrated in Figs. 9 and 10. The results for the dual-microphone models are also included.

As seen in the figures, the general trend of $\mathrm{MSE}_{c}$ and $\mathrm{MSG}_{c}$ is similar, the larger $M$ the smaller $\mathrm{MSE}_{c}$ and the larger $\mathrm{MSG}_{c}$. The slight difference in the pattern between $\mathrm{MSE}_{c}$ and $\mathrm{MSG}_{c}$ is because the optimization of parameters in the models is based on MSE instead of MSG directly.

The reflection model is superior to the other two models, especially when $M$ is between 6 and 21 . In practice, $M$ is usually chosen between 10 and 20 to assure a fast convergence. In this region, the reflection model yields 5-6 dB higher $\mathrm{MSE}_{c}, 5-7 \mathrm{~dB}$ higher $\mathrm{MSG}_{c}$ than the initialization model, and 8-10 dB higher $\mathrm{MSE}_{c}$, and 9-11 dB higher $\mathrm{MSG}_{c}$ than the direct model. To achieve a $25 \mathrm{~dB}^{\mathrm{MSG}}{ }_{c}$, the direct model needs 31 orders and the initialization model needs 16 orders, whereas the reflection model only needs seven reflection replicas of the nominal impulse response. Moreover, including 11 reflection replicas in the reflection model yields around the same $\mathrm{MSG}_{c}$ as the initialization
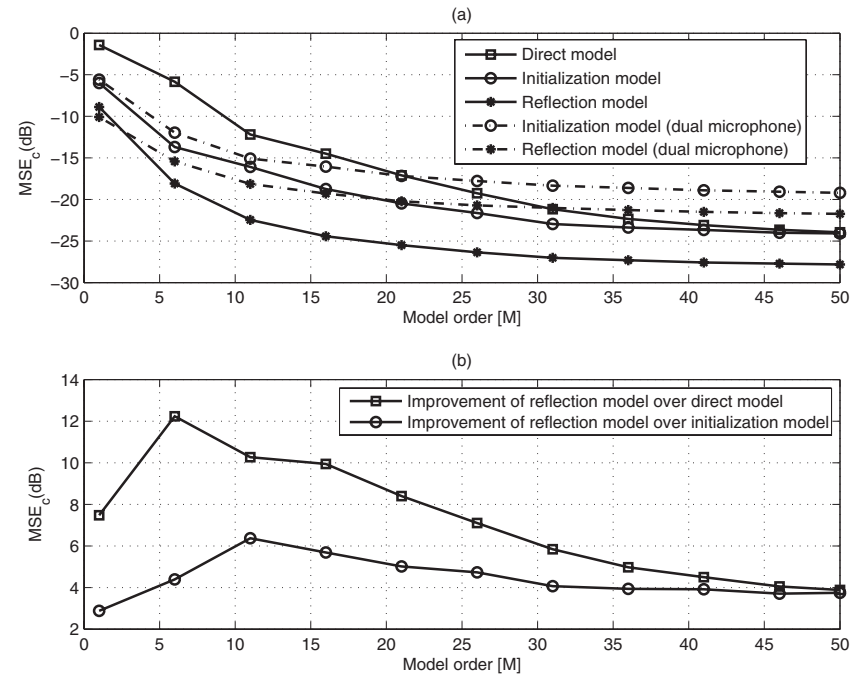

FIG. 9. Comparison of the models for dynamic feedback path modeling in terms of $\mathrm{MSE}_{c}$. (b) shows the $\mathrm{MSE}_{c}$ improvement of the reflection model over direct model and initialization mode. The horizontal axis is the filter length in the direct model and initialization model or the number of reflection components in reflection model.

model with 50-order FIR filter. This shows that 11 reflections are enough to model the dominant characteristics of the dynamic feedback paths resulted from a hand or rigid surface placed close to the hearing aid.

It should be noted that the reflection model does not yield significant improvement when used in the dualmicrophone case. This is because in a complex acoustic environment, the relation between the two feedback paths is very complicated and even more difficult to model than the feedback paths themselves.

\section{CONCLUSIONS AND FUTURE WORK}

This paper describes a novel reflection model for the dynamic feedback path in digital hearing aids. The reflection
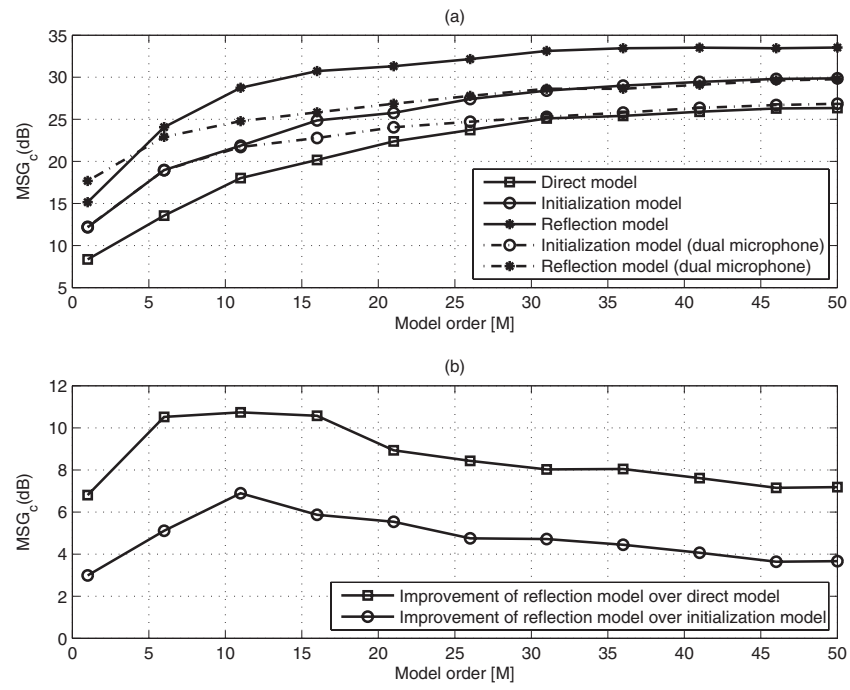

FIG. 10. Comparison of the models for dynamic feedback path modeling in terms of $\mathrm{MSG}_{c}$. (b) shows the $\mathrm{MSG}_{c}$ improvement of the reflection model over direct model and initialization mode. The horizontal axis is the filter length in the direct model and initialization model or the number of reflection components in reflection model. 
properties in addition to the variability of feedback path, when the objects such as a palm or handset are placed close to the hearing aid, have been investigated.

The results based on the measurement of a commercial hearing aid show that the proposed model has a better ability of capturing the dynamic feedback path in these difficult real-life situations and is superior to the existing two models in terms of MSE and MSG. To achieve the same level of MSE or MSG, the number of components required in the proposed model is fewer than the orders of the FIR filter required in the two traditional models.

The results also show the minimum order of the adaptive filter in the two existing models to achieve a certain MSG in the dynamic situations, which could serve as a useful indication in practice for choosing the order of the adaptive filter in feedback cancellation. For the reflection model, 11 reflection replicas are enough to model the dominant characteristics of the proximity effect in dynamic feedback paths.

This paper also investigates the possibility of relating the two feedback paths of a dual-microphone hearing aid for modeling the dynamic feedback paths. It is shown that in a complicated acoustic environment, the relation between the two feedback paths can be very intricate and difficult to exploit to yield better modeling of the dynamic feedback paths.

The future work is to develop an on-line adaptation scheme for this reflection model so that it can improve the performance of feedback cancellation in adverse dynamic situations. The first step to this adaptation scheme is to find a more efficient way of estimating the fractional delays. The frequency-domain approach will be preferred since the implementation of a fractional delay in the time domain requires interpolation. The next step is to find a proper frequency-domain adaptive filtering approach to estimate the dynamic feedback paths based on the estimated delays.

${ }^{1}$ S. F. Lybarger, "Acoustic feedback control," in The Vanderbilt HearingAid Report, edited by Studebaker and Bess (Monographs in Contemporary Audiology, Upper Darby, PA, 1982), pp.87-90.

${ }^{2}$ B. Rafaely, M. Roccasalva-Firenze, and E. Payne, "Feedback path variability modeling for robust hearing aids," J. Acoust. Soc. Am. 107, 26652673 (2000).

${ }^{3}$ J. Kates, "Constrained adaptation for feedback cancellation in hearing aids," J. Acoust. Soc. Am. 106, 1010-1019 (1999).

${ }^{4}$ J. Kates, Digital Hearing Aids (Plural, San Diego, CA, 2008).

${ }^{5}$ J. Hellgren, T. Lunner, and S. Arlinger, "Variations in the feedback of hearing aids," J. Acoust. Soc. Am. 106, 2821-2833 (1999).

${ }^{6} \mathrm{M}$. Stinson and G. Daigle, "Effect of handset proximity on hearing aid feedback," J. Acoust. Soc. Am. 115, 1147-1156 (2004).

${ }^{7}$ D. P. Egolf, B. T. Haley, K. A. Weaver, and D. S. Barker, "The hearing aid feedback path: Mathematical simulations and experimental verification," J. Acoust. Soc. Am. 78, 1578-1587 (1985).

${ }^{8}$ D. P. Egolf, B. T. Haley, H. C. Howell, and S. Legowski, "Simulating the open-loop transfer function as a means for understanding acoustic feedback in hearing aids," J. Acoust. Soc. Am. 85, 454-467 (1989).

${ }^{9}$ K. Nakao, R. Nishimura, and Y. Suzuki, "Calculation of transfer function of acoustic feedback path for in-the-ear hearing aids with a correction for specific acoustic impedance of a tubule," Acoust. Sci. \& Tech. 27, 242244 (2006)

${ }^{10} \mathrm{~J}$. Kates, "Room reverberation effects in hearing aid feedback cancellation," J. Acoust. Soc. Am. 109, 367-378 (2001).

${ }^{11}$ J. Maxwell and P. Zurek, "Reducing acoustic feedback in hearing aids," IEEE Trans. Speech Audio Process. 3, 304-313 (1995).

${ }^{12}$ J. Kates, "Feedback cancellation in hearing aids: A computer simulation results," IEEE Trans. Signal Process. 39, 553-562 (1991).

${ }^{13}$ M. M. A. Spriet, I. Proudler, and J. Wouters, "Adaptive feedback cancellation in hearing aids with linear prediction of the desired signal," IEEE Trans. Signal Process. 53, 3749-3763 (2005).

${ }^{14} \mathrm{~J}$. Hellgren, "Analysis of feedback cancellation in hearing aids with filtered-x $1 \mathrm{~ms}$ and the direct method of closed loop identification," IEEE Trans. Acoust. Speech Signal Process. 10, 119-131 (2002).

${ }^{15}$ R. J. Oliveira, "The active earcanal," J. Am. Acad. Audiol. 8, 401-410 (1997).

${ }^{16}$ G. Ma, F. Gran, F. Jacobsen, and F. Agerkvist, "A new approach for modelling the dynamic feedback path of digital hearing aids," in 2009 IEEE International Conference on Acoustics, Speech and Signal Processing (2009), pp. 209-212.

${ }^{17}$ Y. Jingbo, T. Mengtong, and J. Chang, "Modeling external feedback path of an itedigital hearing instrument for acoustic feedback cancellation," in 2005 IEEE International Symposium on Circuits and Systems (2005), pp. $1326-1329$

${ }^{18}$ J. Ehrenberg, T. Ewart, and R. Morris, "Signal-processing techniques for resolving individual pulses in a multipath signal," J. Acoust. Soc. Am. 63, 1861-1865 (1978).

${ }^{19} \mathrm{R}$. Wu, J. Li, and Z.-S. Liu, "Super resolution time delay estimation via mode-wrelax," IEEE Trans. Aerosp. Electron. Syst. 35, 294-307 (1999).

${ }^{20} \mathrm{~J}$. Li and R. Wu, "An efficient algorithm for time delay estimation," IEEE Trans. Signal Process. 46, 2231-2235 (1998).

${ }^{21} \mathrm{R}$. Brent, Algorithms for Minimization Without Derivatives (Prentice-Hall, Englewood Cliffs, NJ, 1973).

${ }^{22}$ G. Forsythe, M. Malcolm, and C. Moler, Computer Methods for Mathematical Computations (Prentice-Hall, Englewood Cliffs, NJ, 1976).

${ }^{23} \mathrm{M}$. Feder and E. Weinstein, "Parameter estimation of superimposed signals using the EM algorithm," IEEE Trans. Acoust. Speech Signal Process. 36, 477-489 (1988).

${ }^{24}$ Product Data and Specifications of Kemar Manikin Type 45BA, G.R.A.S. Sound \& Vibration, Holte, Denmark (2007).

${ }^{25}$ HI-PRO Hardware Installation Guide, GN Otometrics A/S, Taastrup, Denmark (1993).

${ }^{26}$ L. L. Beranek, Acoustics (McGraw-Hill, New York, 1954). 